\title{
Corticostriatal cells in comparison with pyramidal tract neurons: contrasting properties in the behaving monkey
}

\author{
Erhard Bauswein, Christoph Fromm and Axel Preuss \\ Max-Planck-Institut für biophysikalische Chemie, Abteilung Neurobiologie, Göttingen (F.R.G.)
}

(Accepted 11 April 1989)

Key words: Corticostriatal cell; Motor cortex; Pyramidal tract neuron; Behaving monkey; Antidromic identification; Single-unit recording

\begin{abstract}
Antidromically identified neurons projecting to the putamen (CPNs) and pyramidal tract neurons (PTNs) were recorded from motor and premotor cortex of a monkey which performed a load-bearing task with the wrist. CPNs appeared as a uniform population with very slowly conducting axons and low spontaneous activity. In contrast to PTNs, they exhibited weak, mostly insignificant correlation with graded steady-state forces, responded to torque perturbations with remarkably long latency, and seemed to discharge much later with active movement. Collateral branching of PTNs to the putamen was found to be infrequent (1\%). We suggest that the putamen receives a cortical message that is strikingly different from that sent down the pyramidal tract.
\end{abstract}

The role of the putamen as part of a 'motor' circuit linking the basal ganglia with the neocortex has been evidenced by the demonstration of somatotopically well-organized projections from the sensory and motor cortices ${ }^{8.10,12}$ and by recent findings on the relationship of single cells to specific aspects of motor behavior ${ }^{1-3.11}$ in the primate putamen. However, the functional significance of the corticostriatal part of this 'motor loop' remains obscure, mainly because the actual projection neurons to the putamen have until now not been neurophysiologically identified and studied in any animal preparation. Recent anatomical work stresses the independence of the cells of origin of the various corticofugal systems $^{9}$, thus possibly indicating their functional specialization. Although still a matter of controversy $y^{4.5}$, collateral branching to the striatum of other descending fibers, namely of the pyramidal tract, seems to be negligible in the monkey ${ }^{8.10}$. Other, more general questions posed by our study relate (1) to the issue of columnar organization of the motor cortex, i.e. do neighboring clusters of different types of efferent cell in cortical layer $\mathrm{V}$ constitute a common module ${ }^{9,16}$, and (2) do similarities within a particular corticofugal system transcend areaspecific differences ${ }^{7}$ ? We have therefore compared the properties of antidromically identified corticoputamenal neurons (CPNs) and pyramidal tract neurons (PTNs) recorded from the primary motor cortex (MI) and postarcuate premotor area (PMA) in the behaving monkey.

A fully trained, male rhesus monkey $(8.5 \mathrm{~kg})$ was used for the experiments. Under Nembutal anesthesia and after craniotomy over the left MI-hand-arm region, a recording cylinder (i.d. $18 \mathrm{~mm}$ ) was installed and tilted in the vertical plane, so as to allow transdural microelectrode penetrations perpendicular to the cortical surface. Stereotaxic procedures were aided by the use of X-rays and NMR-tomography. A pair of teflon-insulated stainless steel wires of $150 \mu \mathrm{m}$ diameter (tips exposed over $200 \mu \mathrm{m}$ and vertically separated by $2.5 \mathrm{~mm}$ ) attached to a guide shaft was implanted within the left pyramidal tract (PT) at the most caudal level of the inferior olive. Another, similar bipolar stimulating electrode aimed at the left putamen was inserted

Correspondence: C. Fromm, Max-Planck-Institut für biophysikalische Chemie, Dept. of Neurobiology, Postfach 2841, D-3400 Göttingen, F.R.G. 
through the lateral cortex (L 28) with an angle of $41^{\circ}$ to the vertical axis and $3.9 \mathrm{~mm}$ caudal to the commissura anterior (Fig. 1). Intentionally, we placed the lower electrode tip at the dorsolateral border of the putamen (Put) in order to stimulate, between both tips, the main fiber bundles in the capsula externa where they enter the nucleus ${ }^{8.10}$. Impedances of the implanted electrodes $(40-60 \mathrm{k} \Omega$ ) remained constant throughout the experimental period of 6 months; double shocks delivered via the PT e'ectrode resulted in movement of the contralateral thumb in the awake monkey, at an initial threshold of $0.3 \mathrm{~mA}$ which increased by $25 \%$ during the 6 months. The Evarts' technique (e.g. ref. 6) was used for extracellular recordings with glass-coated $\mathrm{Pt} / \mathrm{Ir}$ electrodes (1-4 M $\Omega$ ). Intracortical microstimulation (ICMS) with a train of 11 cathodal pulses at $300 \mathrm{~Hz}$ was applied through these electrodes, if possible close to the PTNs recorded. We determined the

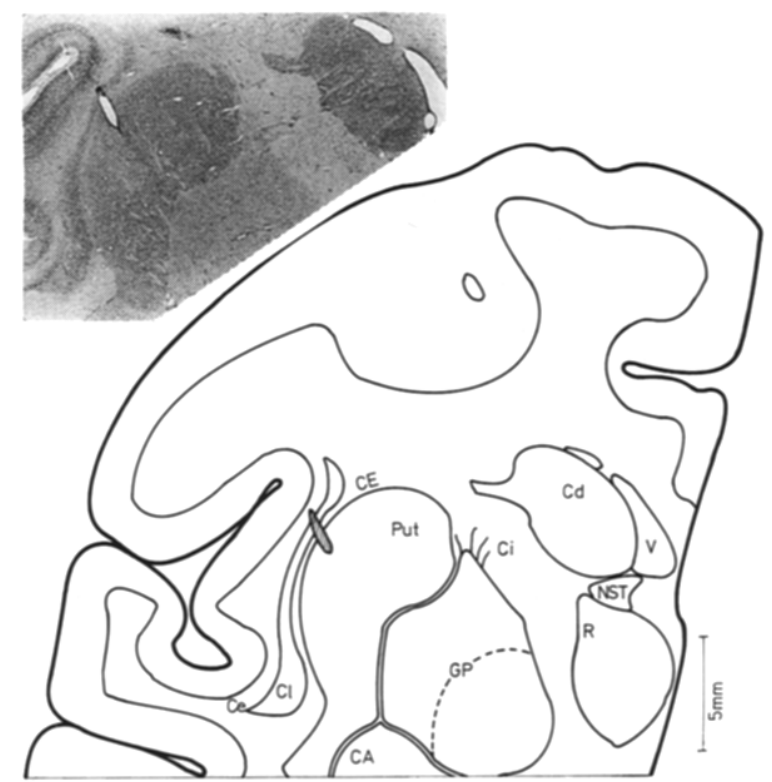

Fig. 1. Projection drawing and corresponding photomicrograph of a frontal section showing the position of part of the chronically implanted electrode array, which was left in the brain during perfusion. The electrode was slightly tilted in the AP direction; only the exact location of the dorsal tip at $\mathrm{Ce}$ is visible here, whereas the deeper wire-tip together with the guide shaft was seen in adjacent sections to invade the putamen by no more than $1.5 \mathrm{~mm}$. Cd, caudatum; CE, capsula externa; $\mathrm{Ce}$, capsula extrema; $\mathrm{Cl}$, claustrum; Put, putamen; $\mathrm{Ci}$, capsula interna; GP, globus pallidus; $\mathrm{CA}$, corpus amygdaloideus; NST, nucleus of stria terminalis; $R$, nucleus reticularis thalami; $\mathrm{V}$, ventriculus lateralis. boundary between PMA and MI according to previously proposed criteria ${ }^{13.15}$, i.e. by an anteroposterior density analysis of Betz cells and by mapping the effects of ICMS, which were absent at sites assigned to PMA, up to a maximal current of $35 \mu \mathrm{A}$.

Fig. 2 shows the antidromic identification of a CPN and the criteria employed: invariance of antidromic latency, $\operatorname{ADL}(\mathrm{A}, \mathrm{B})$, and proper collision interval (C). It was particularly important to regularly ensure the identity of a discriminated unit
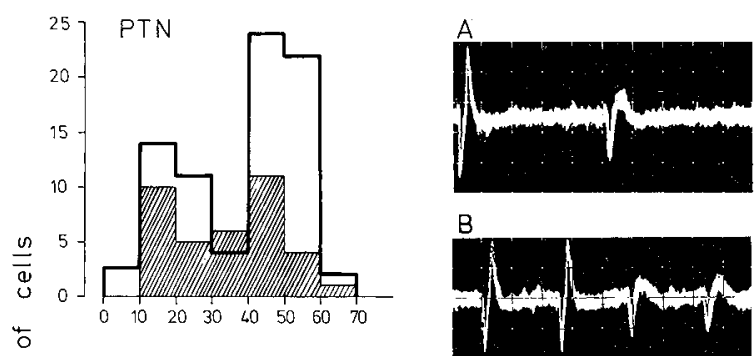

B
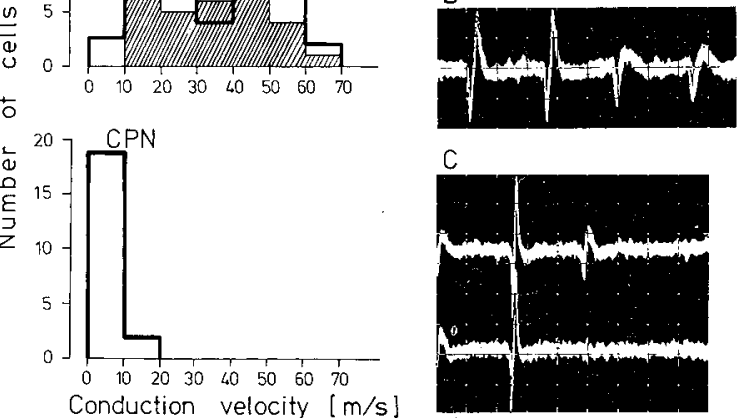

C

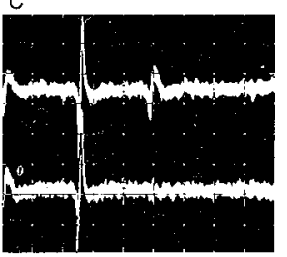

Fig. 2. A-C: antidromic excitation of a cell in MI by stimulation of the ipsilateral putamen. Unit responded at constant latency to four juxtathreshold shocks of $0.7 \mathrm{~mA}$ (A). Double shocks with $2.5 \mathrm{~ms}$ interval (B) and collision test (C) applied at an intensity of $1.3 \times$ threshold. Four superimposed sweeps at $1 \mathrm{~ms} / \mathrm{Div}$. (A,B) and $2 \mathrm{~ms} / \mathrm{Div}$. (C), respectively, for each display. Antidromic latency, ADL, as measured to first deflection of evoked spike, was $4.8 \mathrm{~ms}$ at suprathreshold stimulation. The collision interval in $C$ is $5.1 \mathrm{~ms}$ and defined as the time between the first initial inflection of the spontaneous spike (as deduced from photographs of the complete spike; only its last part being visible at the beginning of the sweeps in C) and the onset of the stimulus (large artifact) which elicited an antidromic response about $50 \%$ of the time. This neuron (same as in Fig. 3C, G,H) did not respond to PT stimulation. On the left, distribution of axonal conduction velocities is shown for 79 PTNs in MI (open bars) and 37 PTNs in PMA (hatched bars) in the upper histogram, and for 21 CPNs in the lower histogram. Conduction velocity was calculated on the basis of the ADL at suprathreshold stimulation and from $\mathrm{X}$-ray and post mortem estimates of the conduction distance. The distance from the putamen site varied between 21-28 $\mathrm{mm}$ depending on the exact recording site of any CPN, while for all PTN axons a global conduction distance of $64 \mathrm{~mm}$ from the PT site to the precentral gyrus was taken. Mean conduction velocity of PTNs in MI $(40.1 \mathrm{~m} / \mathrm{s})$ was significantly different from that of PTNs in PMA $(33.4 \mathrm{~m} / \mathrm{s})$ (Wilcoxon $U$-test, $P<0.05$ ). 
throughout the recording period in the case of the very rarely discharging and small-sized CPNs; thus, the pulse from the amplitude discriminator was fed into the stimulator to trigger the stimulus after the appropriate delay (Fig. 2C). Twenty-one CPNs could be reliably identified and isolated for long enough to allow analysis of task-relations. Their axonal conduction velocities were rather low and narrowly distributed (mean: $6.8 \mathrm{~m} / \mathrm{s}$, range: $3-12 \mathrm{~m} / \mathrm{s}$ ) as compared to those of PTNs (cf. histograms in Fig. 2). From the upper histogram it is also apparent that the PMA (hatched portion) comparatively lacks the more rapidly conducting PTNs that are prominent in MI. CPNs were found over a $6 \times 8 \mathrm{~mm}$ wide area of MI, with two being allocated to PMA, and were encountered on the average $500 \mu \mathrm{m}$ above the PTNs in any given penetration (some being intermingled with and others being up to $900 \mu \mathrm{m}$ higher than the PTNs). Threshold constant-current pulses (duration: $0.25 \mathrm{~ms}$ ) for antidromic activation of CPNs ranged from 0.2 to $1.8 \mathrm{~mA}$ (mean \pm S.D.: $0.8 \pm 0.4 \mathrm{~mA}$ ); in all cases the deeper electrode tip was effective as cathode. None of the $21 \mathrm{CPNs}$ responded to strong PT stimuli of $1.5-3 \mathrm{~mA}$, which were used because $10 \%$ of the PTNs required such high currents for their antidromic invasion. Applying a test and search stimulus of at least $2 \mathrm{~mA}$ at the Put site, one out of 101 tested PTNs could be antidromically driven and shown to give off a collateral. Combined stimulation at both sites and spike occlusion tests on this cell demonstrated a much slower conduction via the collateral branch to the putamen as compared to that of the parent PT axon*.

In contrast to the regular tonic discharge shown by many PTNs, spontaneous activity of CPNs was very low (mean: $2 \mathrm{imp} . / \mathrm{s}$ ), with four units being completely silent during 5-10 min of task performance and two units exhibiting the highest spontaneous firing, between 7 and $17 \mathrm{imp} . / \mathrm{s}$. The monkey performed a position-holding task by grasping a handle that could be rotated by extension-flexion of the right wrist and was coupled to a brushless DC torque motor. The monkey was required to maintain the handle in one of three given wrist positions while different steady torques $(0-0.1 \mathrm{Nm})$ in either direction were applied at varying times and in randomized sequence; correct positioning within the $6^{\circ}$ wide hold zone was indicated by a light. An LSI 11/73 (DEC) computer was used for control of the task, on-line collection of neural and analog data and display. Steady-state firing rate was measured over 1-s periods of postural stability, about 2-3 s after torque change, its mean was computed from at least 15 raster rows at each load level. The Mann-Whitney $U$-test $(P<0.01$ level $)$ was applied to define a statistically significant difference between unit discharges at two different loads (usually for any given unit at the heaviest extensor and flexor loads), as previously described ${ }^{6}$.

Firing rate-load relationships of PTNs were similar to those found in a comparable study on MI PTNs $^{6}$ : either S-shaped functions (in $60 \%$ of cells; e.g. the PTNs in Fig. 3E,F) or roughly linear relations over the range of loads tested (in $40 \%$ ). An equal proportion (45\%) of the PTNs in both areas (31 of 67 tested in MI; 14/31 in PMA) had a significant and monotonic relation of firing rate to load and there was no difference regarding their rate-torque slopes. However, by applying the same statistical criterion (see above), only 2 out of 17 analyzed CPNs exhibited a significant relation to static load (illustrated in Fig. 3A,B). A weak, but for repeated runs consistent trend in 5 other CPNs did not reach this significance-level (e.g. the CPN in Fig. 3D). In 8 penetrations, 10 CPNs could be compared with 14 PTNs in their close vicinity: in 5 loci only the PTNs were load-related (Fig. 3D-F), in one only the CPN showed a significant relation (Fig. 3B), and in two penetrations both types of neuron were unrelated (e.g. Fig. 3C). Occurrence of load-relation in CPNs, unlike in PTNs, was not predictable by the effects of ICMS (see A and C and legend of Fig. 3). In a cortical spot where ICMS resulted in contraction of prime movers for the task (ext. carp. rad. in Fig.

\footnotetext{
*Except for this large antidromic spike (ADL: $1.1 \mathrm{~ms}$ ) which was detectable over $200 \mu \mathrm{m}$, responses to the Put search stimulus commonly appeared above the noise level over a recording distance of only 20-50 $\mu \mathrm{m}$ (in fact we discarded many more units whose single responses between 3 and $10 \mathrm{~ms}$ could not be well isolated), and there was never any antidromic field potential commonly observed at short-latency following PT stimulation. Test stimuli at the Put site also failed to evoke orthodromic pyramidal tract potentials (on recordings from the electrodes in the PT) or any detectable movements (double shocks of $2.2 \mathrm{~mA}$ ). These observations exclude the possibility of current spread to fibers running in the internal capsule.
} 

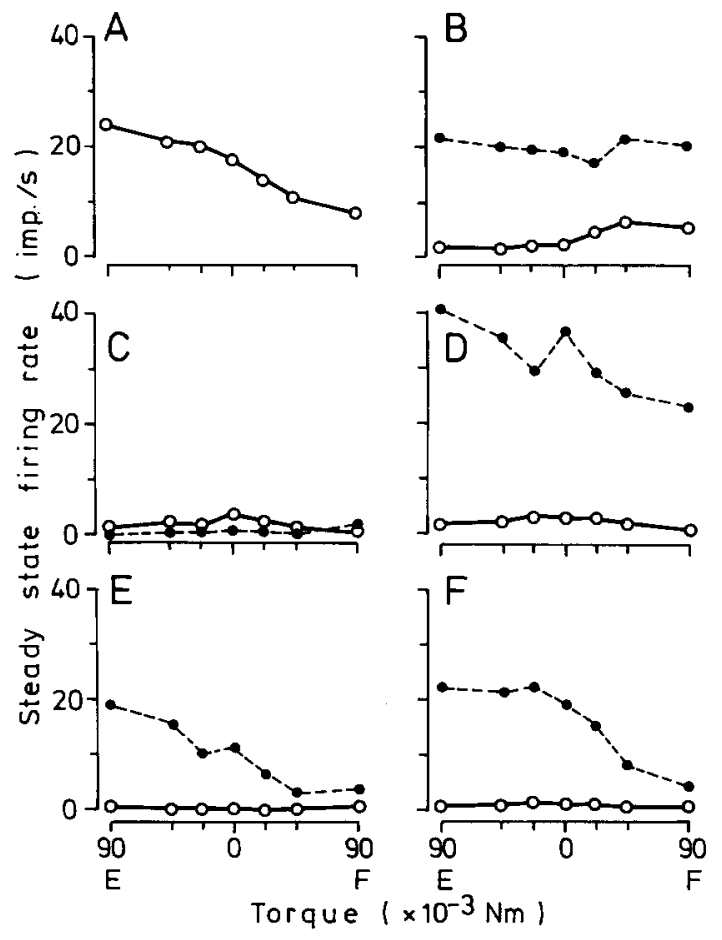
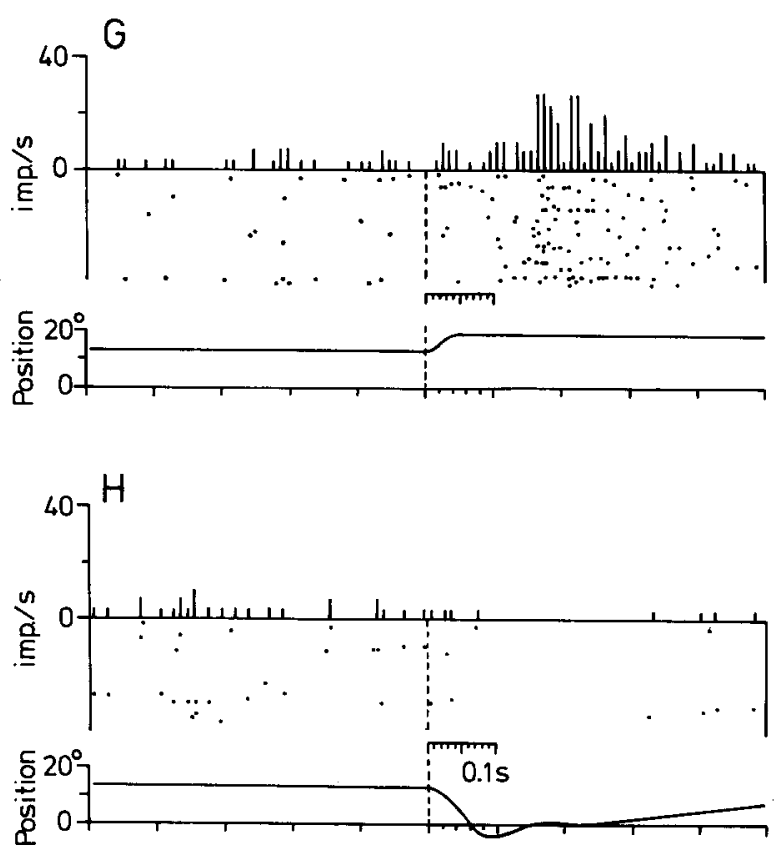

Fig. 3. A-F: firing rate as a function of static torque for pairs of CPN (open circles, solid lines) and PTN (filled circles, dashed) recorded in the same penetrations. Each point represents averaged firing rate over 1-s periods, calculated from at least 15 trials, and measured 2-3 s after torque change during positional maintenance of the wrist against flexor (' $F$ ') or extensor (' $E$ ') loads. Both CPNs in A and B and the 3 PTNs in D-F changed activity significantly with load $(P<0.01$, Mann-Whitney $U$-test). Both types of neurons were either simultaneously recorded (B) or successively over a depth-distance between 50 and $750 \mu \mathrm{m}$ (with the CPNs lying more superficially); no PTN could be recorded in A. ICMS in the vicinity of these neurons yielded lowest-threshold effects in the following muscles: triceps brach. (A,C), brachiorad. (D) biceps brach. (E), and ext. carp. rad. (F); recording in B (negative ICMS) is from the PMA. G-H: Late, directionally specific responses of a CPN to flexion (G) and extension (H) displacements of the wrist caused by torque changes. Torque steps of different size and starting from different steady load levels were applied at the center of the 1-s display (vertical dashed line), on which unit discharge in the form of time histograms (showing average discharge frequency in imp./s for 10-ms bins) and of dot-rasters has been aligned, together with the corresponding averaged position of handle (below).

3F) it was common to find a high percentage of PTNs (and unidentified neurons) being well correlated with load, while the opposite was true for a penetration with ICMS effect in triceps brach. The observation in Fig. 3F is the more important because this CPN was only $50 \mu \mathrm{m}$ distant to the PTN, it phasically discharged during active wrist extensions, but was unrelated to load.

Unit activity was also analyzed in relation to large return movements to the hold zone following torque perturbation and to small corrective movements that the monkey made when the handle drifted from the hold zone. Six CPNs clearly changed activity with large wrist movements and this change was directionally selective. Four of these units also fired intensely with small corrective movements. CPN discharge began near onset of movement or later and lagged onset times of the neighboring PTNs $(n=7)$ by an average of $160 \mathrm{~ms}$.

Nine different torque steps $(0.015-0.113 \mathrm{Nm})$ loading the wrist extensors of flexors resulted in rapid angular displacements $\left(3^{\circ}-30^{\circ}\right)$ of the handle. Twelve CPNs responded to these perturbations, their excitatory responses occurred exclusively for one direction of displacement (Fig. 3G,H). The response latency was remarkably long (mean: 138 $\mathrm{ms}, n=12$ ) as compared to that of PTNs recorded in the same penetrations (mean: $46 \mathrm{~ms}, n=13$ ). In general, PTN responses paralleled the various components of EMG responses observed in many mus- 
cles; PTNs had phasic responses (mean latency: 23 ms, $n=42$ ), which could be correlated with the derivatives of displacement, and/or tonic responses (mean latency: $57 \mathrm{~ms}, n=50$ ) which were related to the size of the new load. CPNs might be best considered in 3 groups. (i) Four units exhibited phasic responses with the shortest latencies (30-70 $\mathrm{ms}$ ), and their responses could be linearly correlated with peak amplitude of displacement. Interestingly, these units did not discharge with active wrist movements. (ii) Three other units were related to active extensions (and partly to static load) and were more tonically activated $50-100 \mathrm{~ms}$ after displacement in the opposite direction (flexion). (iii) Units of the third group responded at even longer latencies. For example, the CPN in Fig. $3 \mathrm{G}$ increased its activity $160 \mathrm{~ms}$ after passive flexion. The response seems to be temporally linked to the perturbation but was not correlated with its kinematic parameters. In Fig. 3G (unlike H, cf. averaged position records) trials have been selected in which the monkey had long reaction times for his return movement to the hold zone in order to demonstrate that this discharge was not associated with execution of active movement. In fact, the direction-specific activity was found to cease $600 \mathrm{~ms}$ before onset of extensions returning the handle. Such an unusual pattern in these few CPNs was not observed in any of the 20 distal and proximal arm muscles recorded from.

The properties of CPNs so far described strikingly resemble those reported for neurons in the putamen $^{1-3,11}$. This is true with respect to the very low spontaneous activity of putamen cells ${ }^{2,3}$, to their rather late discharge with voluntary movement ${ }^{3,11}$ and to their weak relation to steady-state forces. Although there is some disagreement between the two studies as to the frequency of the latter relationship in the putamen ${ }^{3,11}$, it appears that the relation to static force is in principle present in both CPNs and their target cells but much weaker in comparison with PTNs. Like CPNs, putamen cells were shown to respond to passive displacements and to torque perturbations after quite long latencies (mean $=146 \mathrm{~ms}$, with minimum latencies between 40 and $100 \mathrm{~ms})^{11}$; in another study two-thirds of neurons responded above $50 \mathrm{~ms}^{3}$. Another common feature is the directionally specific discharge with active and passive movements. Finally, within the putamen there appear to be 3 functionally different cell-groups: exclusively somatosensory-driven, movement-related ${ }^{2,3,11}$ and set-related neurons ${ }^{1}$ which discharged specifically for the intended direction and during the planning period of forthcoming movements. In our task, the perturbation gives the instruction for the direction of following movement; thus some CPNs of our third group (Fig. 3G,H) might be interpreted to show such a 'set-activity'. It would mean that the functional segregation within the putamen is already reflected in separate input channels from the cortex. This slowly conducting 'motor loop' through the basal ganglia back to the premotor and motor cortices might thus be involved in the control of ongoing movement and in long-term preparation of movements ${ }^{1.3}$.

We found no major functional differences between PTNs recorded from MI and PMA. In contrast, the numerous differences between CPNs and PTNs, especially significant for neurons recorded in the same penetration, were sufficiently clear to warrant a fundamentally different role for the CPN and PTN outputs from MI. A similar conclusion was derived in a previous study in which corticorubral neurons and PTNs were compared ${ }^{7}$. The present results again emphasize a laminar functional differentiation. It seems unlikely that different subsets of corticofugal neurons in close spatial association form a module as defined by common thalamic inputs ${ }^{9,16}$.

While our observations and control tests clearly rule out current spread from the Put stimulation site to the internal capsule which is at least $5 \mathrm{~mm}$ distant, excitation of the corticoclaustral projections from $\mathrm{MI}^{10}$ must be taken into account (Fig. 1). Their origin from layer VI has only been traced in some cortical areas of a few species and is not known for the primate $\mathrm{MI}^{14}$. Nevertheless, CPNs were found to lie mainly in upper cortical layer $\mathrm{V}$, in full accordance with anatomical findings on corticostriatal cells $^{8,9}$, and this fact, together with the rather uniform features exhibited by CPNs, might argue against involvement of corticoclaustral cells. The very low incidence of striatal branching of PTNs $(1 \%)$ in our study, comparing to $6 \%$ in the $\mathrm{cat}^{5}$ and $25 \%$ in the $\mathrm{rat}^{4}$, would indicate a true species difference and is in line with the negative evidence 
for such branching from antero- and retrograde axonal transport studies in the monkey ${ }^{8.10}$. Despite the focal stimulation in our experiment, a more substantial degree of PT collateralization would have shown up, if present, since we would have hardly missed the larger antidromic responses of PTNs during a search involving 135 widely distributed

1 Alexander. G.E.. Selective neuronal discharge in monkey putamen reflects intended direction of planned limb movements, Exp. Brain Res., 67 (1987) 623-634.

2 Alexander. G.E. and DeLong, M.R., Microstimulation of the primate neostriatum. II. Somatotopic organization of striatal microexcitable zones and their relation to neuronal response properties, J. Neurophysiol., 53 (1985) 14171430.

3 Crutcher, M.D. and DeLong, M.R., Single cell studies of the primate putamen. II. Relations to direction of movement and pattern of muscular activity, Exp. Brain Res., 53 (1984) 244-258.

4 Donoghue, J.P. and Kitai, S.T., A collateral pathway to the neostriatum from corticofugal neurons of the rat sensorymotor cortex: an intracellular HRP study, J. Comp. Neurol., 201 (1981) 1-13.

5 Endo, K.. Araki, T. and Yagi, N., The distribution and pattern of axon branching of pyramidal tract cells, Brain Research, 57 (1973) 484-491.

6 Evarts, E.V., Fromm, C., Kroeller, J. and Jennings, A., Motor cortex control of finely graded forces, J. Neurophysiol., 49 (1983) 1199-1215.

7 Fromm, C. . Contrasting properties of pyramidal tract neurons located in the precentral or postcentral areas and of corticorubral neurons in the behaving monkey. In J.E. Desmedt (Ed.), Motor Control Mechanisms in Health and Disease, Advances in Neurology, Vol. 39, Raven, New York, 1983, pp. 329-345.

8 Jones, E.G., Coulter, J.D., Burton, H, and Porter, R., penetrations. For future experiments, implantation of several stimulating electrodes will be necessary to settle this issue.

This work has been supported in part by the Deutsche Forschungsgemeinschaft (Fr 469/6-2).

Cells of origin and terminal distribution of corticostriatal fibers arising in the sensory-motor cortex of monkeys, $J$. Comp. Neurol., 173 (1977) 53-80.

9 Jones, E.G. and Wise, S.P., Size, laminar and columnar distribution of efferent cells in the sensory-motor cortex of monkeys, J. Comp. Neurol., 175 (1977) 391-438.

10 Kuenzle, H., Bilateral projections from precentral motor cortex to the putamen and other parts of the basal ganglia. An autoradiographic study in Macaca fascicularis, Brain Research, 88 (1975) 195-209.

11 Liles, S.L., Activity of neurons in putamen during active and passive movements of wrist, J. Neurophysiol., 53 (1985) 217-236.

12 Liles, S.L. and Updyke, B.V., Projection of the digit and wrist area of precentral gyrus to the putamen: relation between topography and physiological properties of neurons in the putamen, Brain Research, 339 (1985) 245-255.

13 Sessle, B.J. and Wiesendanger, M., Structural and functional definition of the motor cortex in the monkey (Macaca fascicularis), J. Physiol. (Lond.), 323 (1982) 245-265.

14 Sherk, H., The claustrum and the cerebral cortex. In E.G. Jones and A. Peters (Eds.), Sensory-Motor Areas and Aspects of Cortical Connectivity, Cerebral Cortex, Vol. 5, Plenum, New York, 1986, pp. 467-499.

15 Weinrich, M. and Wise, S.P., The premotor cortex of the monkey, J. Neurosci., 2 (1982) 1329-1345.

16 Wiesendanger, M., Redistributive function of the motor cortex, Trends Neurosci., 9 (1986) 120-125. 